\title{
Clinicopathological and immunohistochemical characteristics of pigmented oral squamous cell carcinoma
}

\author{
CUONG MINH TRAN ${ }^{1}$, TAKESHI KUROSHIMA ${ }^{1}$, YU OIKAWA ${ }^{1}$, YASUYUKI MICHI ${ }^{1}$, \\ KOU KAYAMORI ${ }^{2}$ and HIROYUKI HARADA ${ }^{1}$ \\ Departments of ${ }^{1}$ Oral and Maxillofacial Surgery, and ${ }^{2}$ Oral Pathology, Graduate School of Medical and Dental Sciences, \\ Tokyo Medical and Dental University, Bunkyo-ku, Tokyo 113-8549, Japan
}

Received November 1, 2020; Accepted January 12, 2021

DOI: $10.3892 / 01.2021 .12600$

\begin{abstract}
Pigmented oral squamous cell carcinoma (POSCC) is a rare and underrecognized pathological variant of oral squamous cell carcinoma (OSCC). The current study aimed to evaluate the clinicopathological characteristics, treatment outcomes and prognosis of patients with POSCC and to investigate its oncological properties using immunohistochemical studies. A total of 1,512 patients were pathologically diagnosed with squamous cell carcinoma of the oral cavity, and were treated at the Department of Oral and Maxillofacial Surgery, Tokyo Medical and Dental University between January 2001 and December 2018. A total of 25 patients had POSCC and underwent radical surgery. Of these 25 patients, 23 presented with early $\mathrm{T}$ stage disease. Additionally, 22 patients were negative for cervical lymph nodes metastasis. Only one patient had local recurrence. The 5-year disease-free and disease-specific survival rates were 86.6 and $95.8 \%$, respectively. Immunohistochemically, a high percentage of POSCC exhibited low p53 and Ki-67, preserved E-cadherin or negative vimentin expression. The results suggested that POSCC tends to exhibit non-aggressive oncological behavior and demonstrates a good prognosis.
\end{abstract}

\section{Introduction}

Oral cancer is a common malignancy worldwide, with an estimated 53,260 new cases and 10,750 deaths in 2020 (1). Oral squamous cell carcinoma (OSCC), which accounts for more

Correspondence to: Dr Takeshi Kuroshima, Department of Oral and Maxillofacial Surgery, Graduate School of Medical and Dental Sciences, Tokyo Medical and Dental University, 1-5-45 Yushima, Bunkyo-ku, Tokyo 113-8549, Japan

E-mail: kuroosur@tmd.ac.jp

Abbreviations: SCC, squamous cell carcinoma; OSCC, oral squamous cell carcinoma; POSCC, pigmented oral squamous cell carcinoma; EMT, epithelial-mesenchymal transition

Key words: oral squamous cell carcinoma, pigmentation, p53, Ki-67, E-cadherin, vimentin than $90 \%$ of all malignant tumors of the oral cavity, is mainly treated by surgery, radiotherapy, and adjuvant chemotherapy. The prognosis in patients with early-stage OSCC has substantially improved (2). However, the overall survival rate in patients with advanced OSCC has not improved significantly during the past four decades (3). New approaches to early detection, risk assessment, and early intervention are ongoing to improve the survival in patients with OSCC. Therefore, studies elucidating the oncological behavior of OSCC are needed.

The presence of pigmented melanocytes in non-melanocytic neoplasms has been documented in various entities in several sites, such as neuroendocrine carcinoma (4), breast carcinoma (5), and salivary gland tumor (6). Squamous cell carcinoma (SCC) occasionally shows pleomorphic histological forms with several subtypes. Among them, SCC with proliferating dendritic melanocytes is a rare entity that has been reported in the skin (7), uterine cervix (8), conjunctiva (9), nasal cavity (10), scrotum (11), and external auditory canal (12). The clinical course of pigmented SCC in these regions is nearly the same as that of nonpigmented SCCs, and the prognosis is similar to that of the conventional SCC (7,10-12).

Although pigmentation, commonly found in the oral mucosa, represents various clinical patterns ranging from physiological alterations to oral manifestations of systemic diseases and malignancies, pigmented oral squamous cell carcinoma (POSCC) is a rare and underrecognized pathological variant of OSCC. To the best of our knowledge, only 18 cases have been reported in the English literature (13-22). Owing to the lack of a large case series, the clinical characteristics, treatment outcomes, and prognosis of POSCC remain unclear.

Several types of biological markers have been used to investigate the biological and clinical behavior of OSCC and head and neck SCC. However, only a few reports on the use of these biological markers for POSCC are available. p53 is a tumor suppressor gene that regulates cell cycle control, apoptosis, and preservation of genetic stability (23). In head and neck SCC, p53 mutations acquire oncogenic functions to promote tumorigenesis and tumor progression (24). Ki-67 is a potential marker that reflects the total fraction of cell growth in a neoplasm and serves as a molecular indicator of survival and recurrence (25). High Ki-67 expression is a 
negative prognostic marker in patients with OSCC, especially in Asian populations (25). E-cadherin and vimentin are useful indicators of the epithelial-mesenchymal transition (EMT), a critical biological event in which cohesive and polarized epithelial cells switch to mesenchyme-like cells exhibiting no polarization and high mobility (26). This acquired migratory phenotype is essential for tumor growth, particularly during tumor invasion and metastasis. A previous study showed that overexpression of EMT factors was significantly associated with a poor prognosis and could serve as a prognostic factor for head and neck SCC (26). Despite the great interest in the potential malignancy and pathophysiology of POSCC, there have been no studies on the biological properties of these molecules in POSCC because of the rarity of disease occurence.

Therefore, this study aimed to evaluate the clinicopathological characteristics, treatment outcomes, and prognosis in patients with POSCC and to investigate the oncological properties of POSCC via immunohistochemical analysis of p53, $\mathrm{Ki}-67$, E-cadherin, and vimentin expression.

\section{Materials and methods}

Patients and tissue specimens. We retrospectively evaluated all patients diagnosed as having pathologically-confirmed SCC of the oral cavity, who were treated at the Department of Oral and Maxillofacial Surgery, Tokyo Medical and Dental University between January 2001 and December 2018. Pigmented lesions were observed in 26 out of the 1,512 patients. One patient with metal-derived pigmentation, which was determined using the bleaching method, was excluded. Therefore, a total of 25 patients (1.7\% of all patients) were included in this study. Clinical data on age, sex, primary location of the tumor, TNM classification, stage, treatment, surgical margins, pathological cervical lymph node metastases, locoregional recurrence, and prognosis were obtained from their medical records. Clinical staging was based on the TNM staging system of the Union for International Cancer Control, 7th edition (27). The patterns of invasion in the samples were analyzed according to the method used by Anneroth et al (28) and subsequently classified as either low-invasive OSCC (patterns of invasion grades 1, 2, or 3) or high-invasive OSCC (pattern of invasion grade 4) (29). The patients were followed up for a median of 1,789 days (interquartile range, 78-4372).

The specimens were fixed in $10 \%$ formalin, embedded in paraffin, and cut into $4-\mu \mathrm{m}$ sub-serial sections. All hematoxylin and eosin-stained surgical slides were reviewed, and representative blocks were selected for each patient. The sections were processed with a bleaching method using potassium permanganate to ensure that the pigments were melanin (30). Furthermore, we assessed whether the melanocyte loading was opposite to the submucosal inflammation. Submucosal inflammation surrounding the pigmented and nonpigmented areas was evaluated via hematoxylin and eosin staining based on inflammatory infiltration rich in lymphocytes and neutrophils.

Immunohistochemistry. The paraffin blocks of the specimens were cut into $4-\mu \mathrm{m}$ sections and examined immunohistochemically. The sections were deparaffinized with xylene and rehydrated through a series of graded alcohol concentrations, following which they were transferred and rinsed with phosphate-buffered saline (PBS) (Dako Denmark A/S). The epitopes were then retrieved by heating the samples in $0.01 \mathrm{M}$ citrate buffer ( $\mathrm{pH}$ 6.0) for $30 \mathrm{~min}$. After cooling, endogenous peroxidase activity was blocked by $3 \%$ hydrogen peroxide in methanol for $30 \mathrm{~min}$. The slides were washed three times with PBS and incubated overnight at $4^{\circ} \mathrm{C}$ with monoclonal mouse antibody HMB45 (dilution 1:100; Dako), monoclonal mouse anti-human Melan-A (dilution 1:50, clone A103; Dako), monoclonal mouse anti-human p53 (dilution 1:100, clone DO-7; Dako), monoclonal mouse anti-human Ki-67 (dilution 1:100, clone MIB-1; Dako), monoclonal mouse anti-human E-cadherin (dilution 1:500, clone $\mathrm{NCH}-38$; Dako), or vimentin (RP21) rabbit monoclonal antibody (dilution 1:100, Cell Marque). The reactive products were detected using a Histofine SAB-PO Kit (Nichirei Biosciences), followed by the assessment of color development using 3,3'-diaminobenzidine as a chromogen and Giemsa staining for HMB-45 and Melan-A slides, along with Mayer's hematoxylin as a counterstain for the remaining groups. Appropriate positive control sections were processed in parallel. The specificity of staining was checked in negative control slides in which a buffer solution substituted the primary specific antibodies.

The sections were analyzed by two independent observers who were blinded to the clinical data and outcomes. Doubtful cases were reassessed, and discrepancies were settled by consensus. Protein expression was semiquantitatively determined by visual assessment under a light microscope in three representative fields on each slide (Olympus System Microscope Model BX43). Image acquisition was performed with a digital camera (AdvanCam-U3II; Advan Vision Co.) coupled to the microscope.

For HMB-4 and Melan-A staining, a brown cytoplasmic and membranous stain was counted as positive (31). For p53 and Ki-67 staining, the result was considered positive when a brown color appeared in the cell nuclei. The proportion of positively stained cells was expressed as a percentage $(0-100 \%)$ at the invasive tumor front. Low and high levels of p53 expression were defined as immunostaining in $<50 \%$ and $\geq 50 \%$ of epithelial tumor cells, respectively (24). Subsequently, p53 expression was classified as normal (low expression) and abnormal (no or high expression). Meanwhile, Ki-67 expression was classified as low and high if immunostaining occurred in $<20 \%$ and $\geq 20 \%$ of epithelial tumor cells, respectively (25). Staining for E-cadherin (29) was classified as preserved and reduced if membranous immunostaining occurred in $>50 \%$ and $\leq 50 \%$ of epithelial tumor cells, respectively. The expression of vimentin (29) was classified as negative and positive if cytoplasmic immunostaining occurred in $<10 \%$ and $\geq 10 \%$ of epithelial tumor cells, respectively.

Statistical analyses. Survival analyses were conducted using the Kaplan-Meier method. Disease-specific survival was measured from the date of surgery to the date of death from uncontrolled POSCC. Disease-free survival was measured from the date of surgery to the date of relapse. The association between p53 and Ki-67 expressions was analyzed using the Fisher's exact test. The log-rank test was used to compare differences in survival between the two groups. All statistical 
Table I. Clinicopathological characteristics of patients $(n=25)$.

Variables Number

Age, years
Median (range)
Sex
Male
Female

Female

Primary site

Tongue

Retromolar trigone

Buccal mucosa

Floor of the mouth

Gingiva

Surface configuration

Exophytic

Ulcerative

Indurated

Leukoplakic/Erythroplakic

pT stage

T1-T2

T3-T4

Differentiation

Well

Moderate

Poor

Pathological cervical lymph node metastases

Negative

Positive

Pattern of invasion

Low

High

Surgical margin

Negative

Positive

PORT

No

Yes

Locoregional recurrence

Negative

Positive

Prognosis

NED

AWD

DOD

DOC

PORT, postoperative radiotherapy; NED, no evidence of disease; AWD, alive with disease; DOD, dead of disease; DOC, dead of other causes.

analyses were performed using JMP14 (SAS Institute). A P-value of $<0.05$ was considered statistically significant.

\section{Results}

Clinicopathological findings and treatment outcomes. The median age of the patients was 62 (range, 26-85) years, and there were 13 male and 12 female patients. Table I summarizes the clinicopathological characteristics of patients. The most common location of POSCC was the tongue $(n=18,72 \%)$, followed by the retromolar trigone $(n=3,12 \%)$, buccal mucosa $(n=2,8 \%)$, floor of the mouth $(n=1,4 \%)$, and gingiva $(n=1,4 \%)$. All pathological $\mathrm{T}$ stages of the disease were observed, and $23(92 \%)$ tumors were diagnosed as T1-T2. The surface configuration of tumors comprised exophytic $(n=6,24 \%)$, ulcerative $(n=5,20 \%)$, indurated $(n=7,28 \%)$, and leukoplakic or erythroplakic $(n=7,28 \%)$ types. With regard to differentiation, $20(80 \%)$ tumors were classified as well differentiated, $4(16 \%)$ as moderately differentiated, and $1(4 \%)$ as poorly differentiated. Regarding the pattern of invasion, 13 samples were initially classified as grade 1, 8 as grade 2, 2 as grade 3, and 2 as grade 4 . Subsequently, these scores were dichotomized as $23(92 \%)$ low-invasive and $2(8 \%)$ high-invasive OSCCs. Three patients developed cervical lymph node metastases and underwent neck dissection.

All patients underwent surgery, and one patient was administered preoperative chemotherapy (two courses of cisplatin and 5-fluorouracil). One patient developed local recurrence of POSCC. This patient underwent salvage surgery and was alive without disease at the last follow-up. Two of the three patients with pathological cervical lymph node metastases had regional recurrences. One of these patients was alive with disease at the last follow-up, however, and the other one had died of uncontrolled regional recurrence. Overall, 3 of the 25 patients had locoregional recurrence during the follow-up period. There were no distant metastases observed.

Fig. 1 shows the Kaplan-Meier estimates of the 5-year disease-free survival $(86.6 \%)$ and disease-specific survival $(95.8 \%)$ rates.

Characteristics of the pigmentations of POSCC. Pigmentation was observed macroscopically within the tumor and surrounding the mucosal lesion. On histopathological examination, $18(72 \%)$ patients showed pigmentation within the OSCC, while 7 (28\%) patients showed pigmentation within the leukoplakia associated with the tumor. The pigmentation showed wide-ranging features, with asymmetrical and scattered distribution, uneven color intensity, and ill-defined borders.

Microscopically, the histopathological features were proliferation of atypical squamous cells, which was similar to that in conventional SCC, with intermingled melanin without atypia. Immunohistochemical staining of the tumor with HMB45 and Melan-A showed strong positivity within the melanin pigments (Fig. 2). These melanin pigments were not uniformly distributed and were dispersed in the cytoplasm of neoplastic keratinocytes, the intercellular spaces from the surface to the basal layer of the epithelium, or occasionally within the superficial portion of the lamina propria. Morphologically, three types of melanin were recognized: dendritic melanocytes, pigment blockage melanocytes, and melanophages. Melanocyte colonies were commonly formed by pigment blockade melanocytes, whereas dendritic melanocytes were uniformly distributed without colonization. 
A

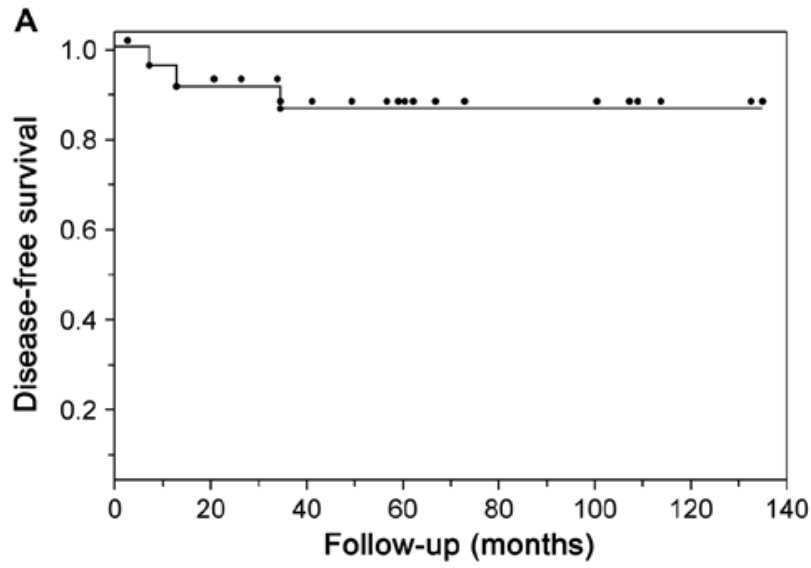

B

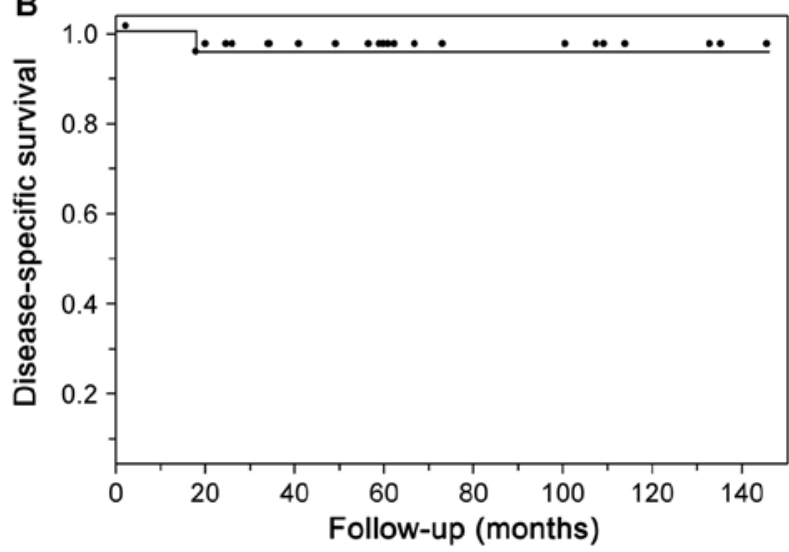

Figure 1. Kaplan-Meier plots of 25 patients with pigmented oral squamous cell carcinoma. (A) 5-year disease-free survival (86.6\%) and (B) 5-year disease-specific survival $(95.8 \%)$.

Table II. Immunohistochemical expression $(\mathrm{n}=25)$.

\begin{tabular}{lc}
\hline Marker & $\mathrm{N}(\%)$ \\
\hline p53 & \\
Low & $18(72)$ \\
High & $7(28)$ \\
Ki-67 & \\
Low & $19(76)$ \\
High & $6(24)$ \\
E-cadherin & \\
Preserved & $24(96)$ \\
Reduced & $1(4)$ \\
Vimentin & \\
Negative & $24(96)$ \\
Positive & $1(4)$ \\
\hline
\end{tabular}

Notably, 7 (28\%) tumors showed early invasion patterns, with architectural disturbance of the stratified squamous epithelium. Increased mitotic figures and drop-shaped rete ridges with no invasive growth toward subepithelial tissues were also noted in these tumors.

All patients had intense $(n=21,84 \%)$ or vague $(n=4,16 \%)$ inflammatory infiltration rich in lymphocytes and neutrophils in the submucosa facing the pigmented area with localization of melanocytes in POSCC. In contrast, in the nonpigmented area of POSCC, there was intense inflammatory infiltration in $11(44 \%)$, vague inflammatory infiltration in $1(4 \%)$, and minimal inflammatory infiltration in $13(48 \%)$ patients (Fig. 3).

Immunohistochemical studies of p53, Ki-67, and EMT-associated molecules. Table II and Fig. 4 show the immunohistochemical expression of p53, Ki-67, and EMT-associated molecules and their representative staining. Overall, a high percentage of POSCC cases exhibited low expression of $\mathrm{p} 53$ and $\mathrm{Ki}-67$, preserved expression of E-cadherin, and negative expression of vimentin. Specifically, p53 and Ki-67 were identified in 18 (72\%) and 19 (76\%) cases, respectively, with a low
Table III. p53 and Ki-67 expression.

\begin{tabular}{lrc}
\hline & \multicolumn{2}{c}{ p53 expression } \\
\cline { 2 - 3 } Ki-67 expression & Low & High \\
\hline Low & 14 & 5 \\
High & 4 & 2 \\
\hline
\end{tabular}

Fisher's exact test, two-tailed $\mathrm{P}>0.99$.

expression index. There was no case with no expression of p53 in tumor cells of POSCC. There was no statistically significant association between $\mathrm{p} 53$ and Ki-67 staining (Fisher's exact test, two-tailed P-value>0.99) (Table III). Furthermore, there were no statistical differences in the DFS (log-rank test, $\mathrm{P}=0.27$ or $\mathrm{P}=0.78$ ) and $\mathrm{DSS}$ (log-rank test, $\mathrm{P}=0.52$ or $\mathrm{P}=0.56$ ) between low- and high- p53 or Ki-67 expression groups, respectively (Fig. 5). Preserved E-cadherin expression was detected in $24(96 \%)$ tumors, and positive expression of vimentin was observed in 1 (4\%) tumor. EMT status was assessed based on the expression of both proteins. Accordingly, 24 (96\%) tumors exhibited preserved E-cadherin and negative vimentin expressions, and 1 (4\%) showed reduced E-cadherin and positive vimentin expressions.

\section{Discussion}

POSCC is a rare variant of SCC that is histologically similar to typical SCC but with the presence of non-neoplastic melanocytes within the lesion $(20,22)$. Pigmentation in POSCC is not always macroscopically visible and may be detected only microscopically $(18,19)$. Our study examined only the cases in which the pigmentation could be macroscopically observed in the primary sites. There was no data in the literature on the prevalence of POSCC. In this study, POSCC was prevalent in $1.7 \%$ of the total OSCC cases. To the best of our knowledge, this is the first study to evaluate the clinicopathological and biological features of POSCC. 
A

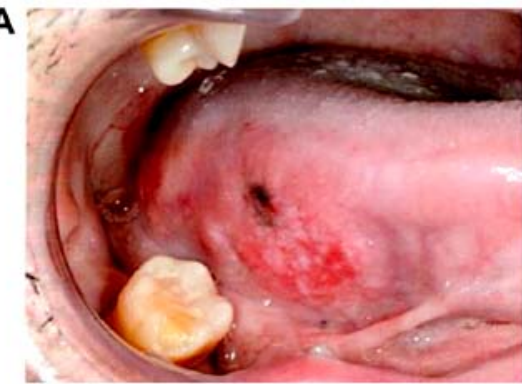

C

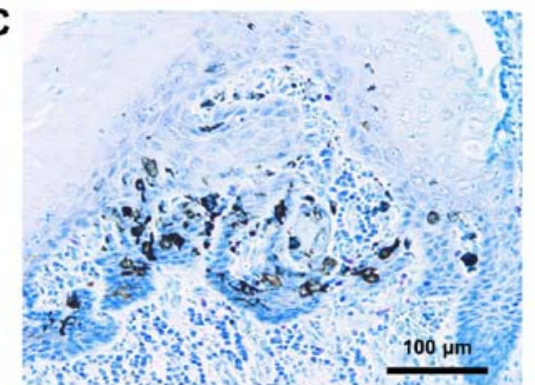

E

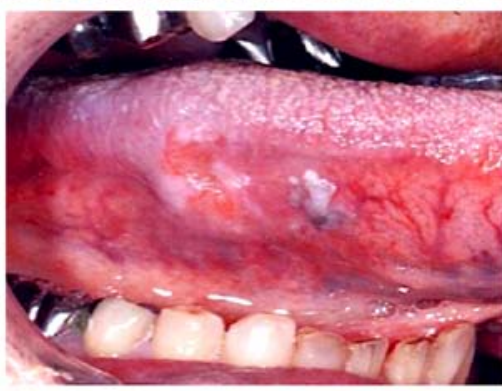

G

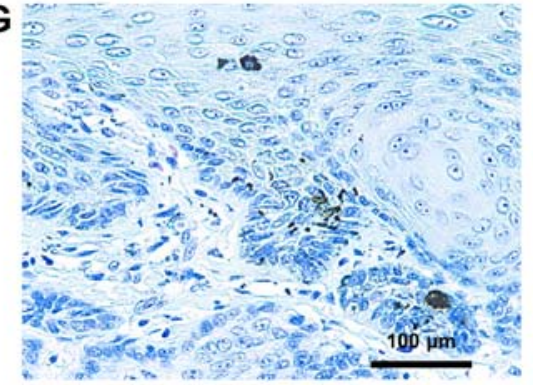

B

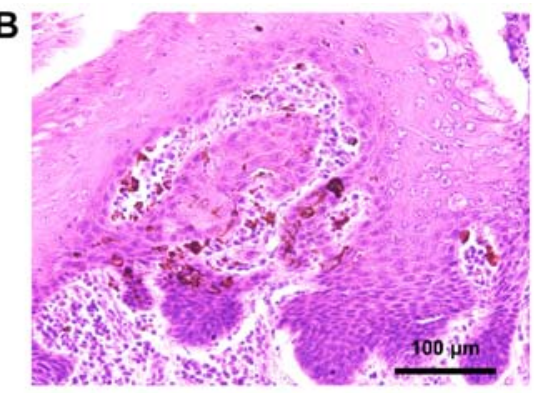

D

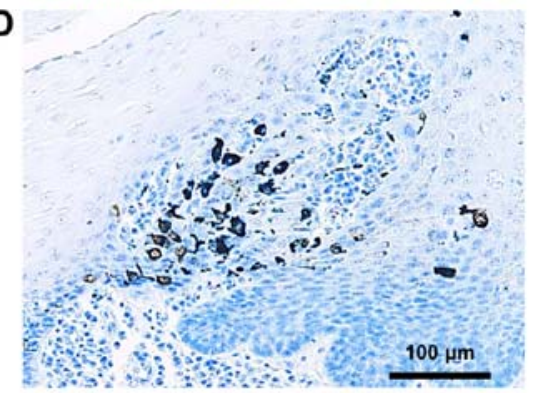

F

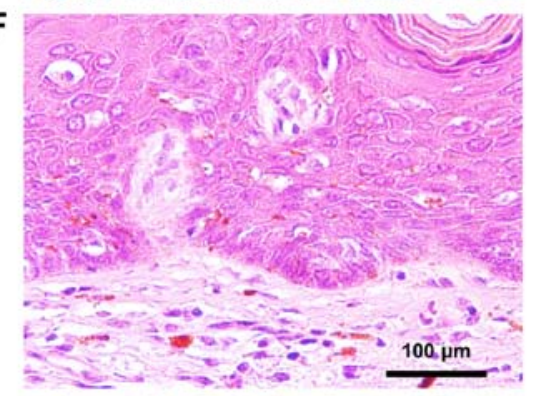

H

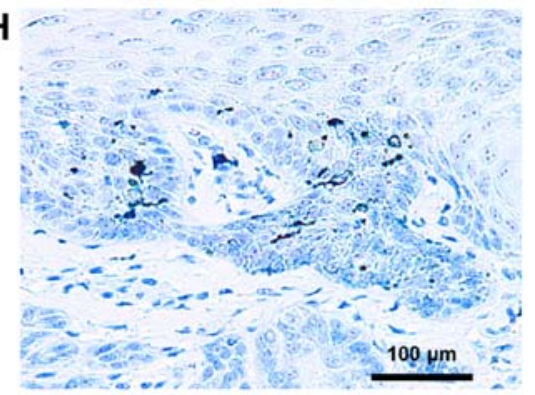

Figure 2. Macroscopic and microscopic results of pigmentation in pigmented oral squamous cell carcinoma. Clinical presentations revealed macro distributions of pigmentation within the (A) tumor and (E) leukoplakia. Microscopic arrangement of melanin in hematoxylin and eosin staining, HMB45, and Melan-A immunoreaction within the tumor (B, C and D) and leukoplakia (F, G and H), respectively (magnification, $\mathrm{x} 200$ ).

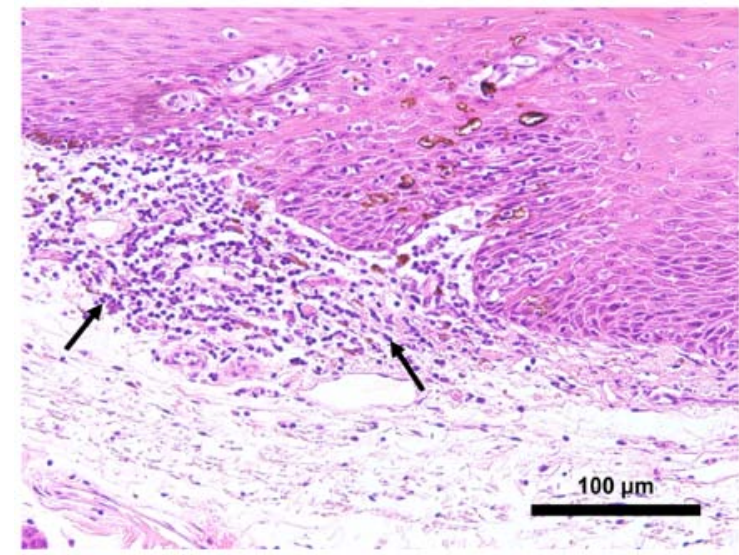

Figure 3. Inflammatory infiltration near pigmented areas. Microscopic photo exhibited a dense inflammatory cell infiltration with lymphocytes and neutrophils in the submucosa (arrows) compared with adjacent non-pigmented carcinoma (magnification, $\mathrm{x} 100)$.
The most common location of POSCC in this study was the tongue $(72 \%)$, which is consistent with previous findings (13-22) and in line with conventional OSCC. Similar to 18 reported cases (13-22), 23 tumors (92\%) were diagnosed as pathological T1-T2 stage. In total, 23 of 25 cases (92\%) were low-invasive type POSCC. There were 3 of 25 patients (12\%) who had pathological lymph node metastases, compared with 1 in the previously 18 reported cases (5.6\%) (20). Cervical lymph node metastases generally develop in $30 \%$ of conventional OSCC cases (32). Although the number of cases in our study were insufficient to draw any conclusions, these results suggest that POSCC has an early tumor stage with a low tendency of metastasis to the cervical lymph nodes. There was 1 POSCC case (4\%) with local recurrence in our study, compared with that of 1 among 18 previously reported cases (5.6\%) (20). Further, we found a 5-year disease-free and disease-specific survival rates of 86.6 and $95.8 \%$, respectively, in the POSCC 


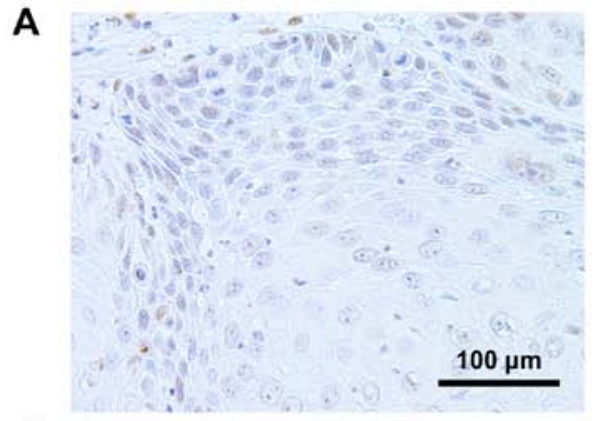

C

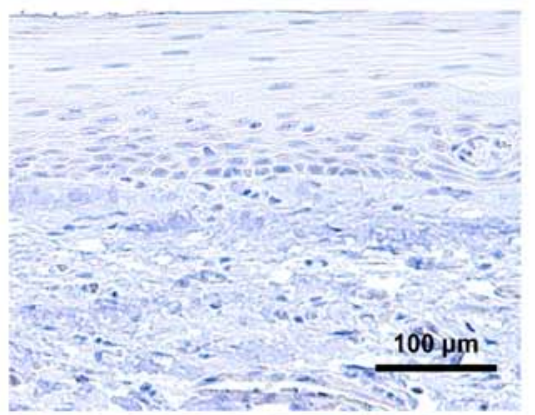

E

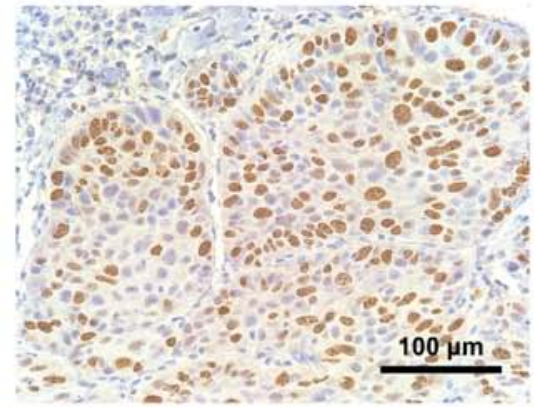

G

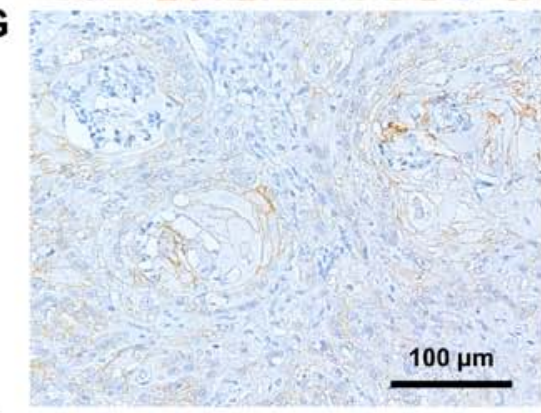

I

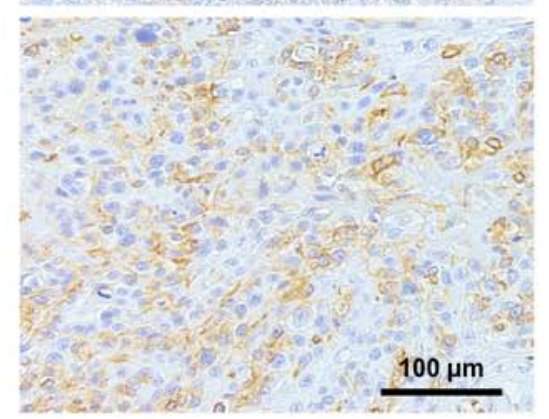

B

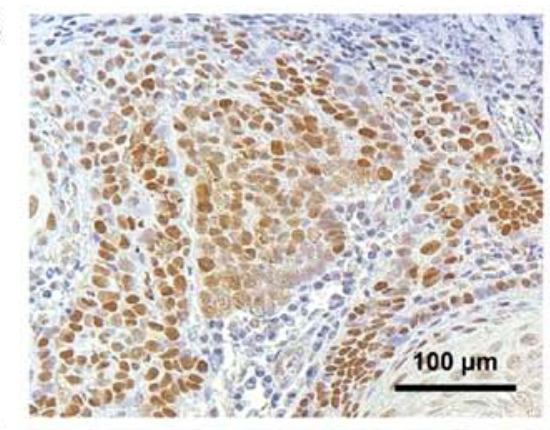

D

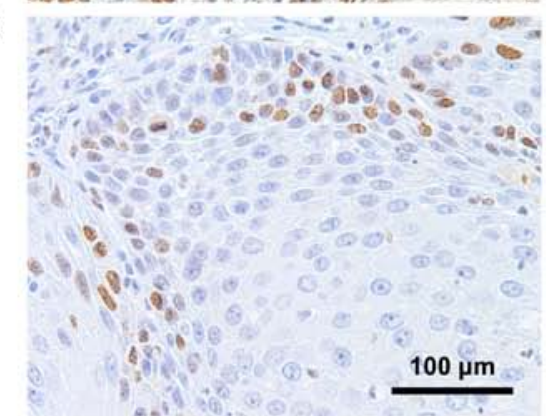

$\mathbf{F}$

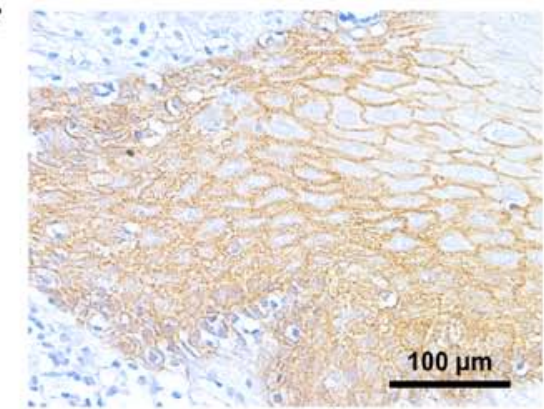

H

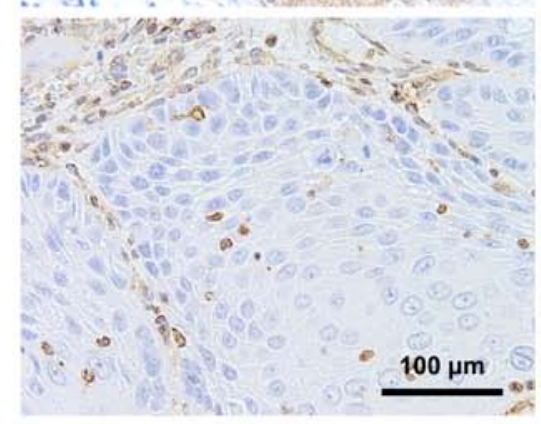

Figure 4. Immunohistochemical staining showing low and high expressions of (A and B) p53, (D and E) Ki-67, and (C) normal expression of p53. Images demonstrating $(F)$ preserved and $(\mathrm{G})$ reduced E-cadherin expression. Images demonstrating $(\mathrm{H})$ negative and (I) positive expression of Vimentin (magnification, x200).

patients. These results suggest that the patients with POSCC exhibit good local control and good prognosis.

Our study found that pigments were interspersed in neoplastic cells from the epithelial surface to the basal layer. Histological analysis revealed that $28 \%$ of cases, showing early invasion patterns, shared some similarities with the characteristics of pigmented carcinoma in situ (22). In previous studies, there have been four cases of OSCC in situ with the symbiosis of melanocytes $(13,15,21,22)$, two of which showed white discoloration on the lesion $(15,21)$. Our study found pigmentation in leukoplakia associated with the tumor in 7 cases $(28 \%)$. Leukoplakia is one of the most common 
A

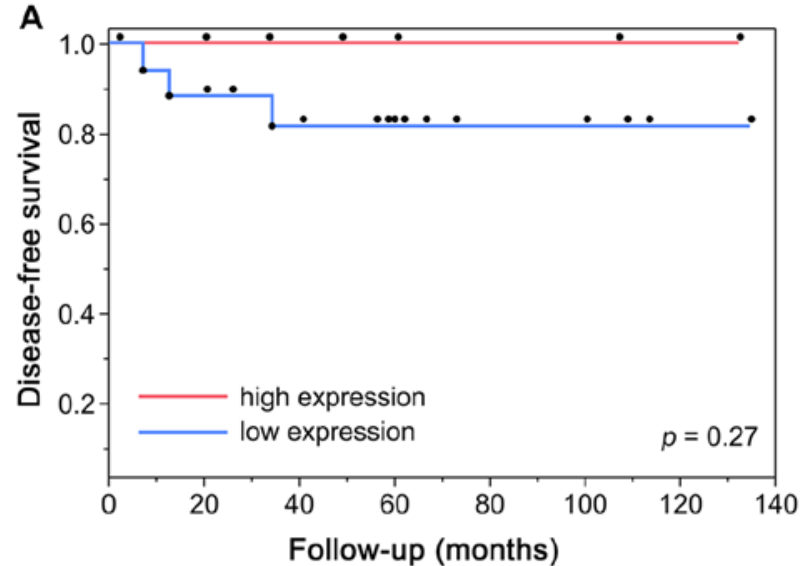

C

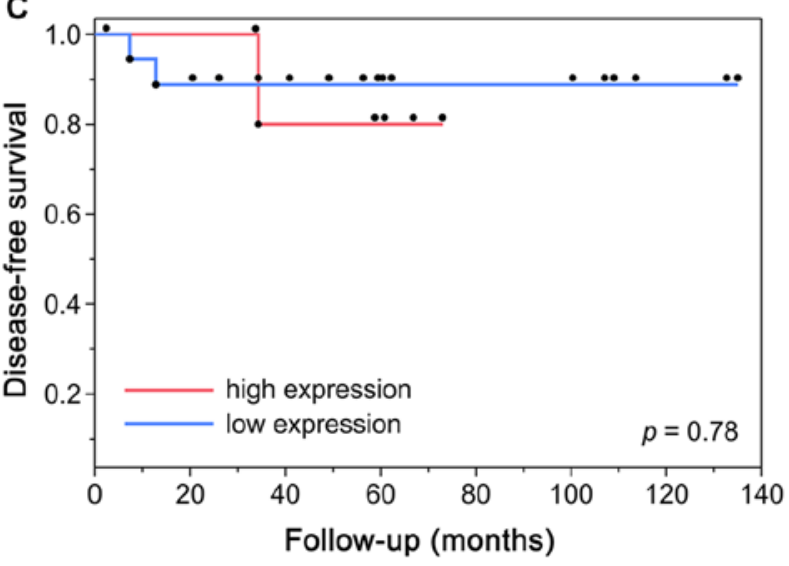

B

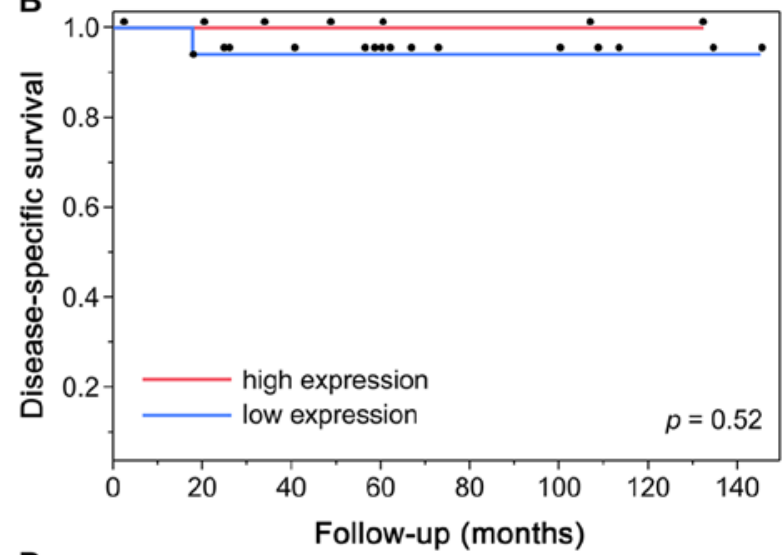

D

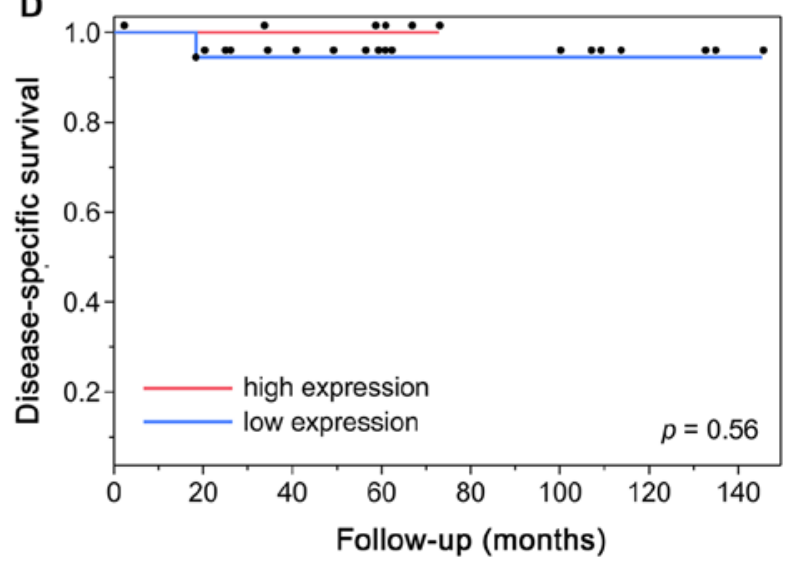

Figure 5. Correlation of protein expression levels with the prognosis of patients with pigmented squamous cell carcinoma. Kaplan-Meier survival curves and log-rank tests between low and high (A and B) p53 or (C and D) Ki-67 expression groups in terms of disease-free survival and disease-specific survival, respectively.

potentially malignant disorders of the oral cavity (33). Matsumoto et al (21) stated that the presence of a pigment in a white mucosal lesion might be a sign of a pigmented pre-cancerous lesion or pigmented SCC. The appearance of pigmentation may not be an incidental physiological finding but is probably reactive and stimulated by local microenvironmental triggers from SCC tumors. It has been reported that the enhanced expression of various growth factors and cytokines in carcinoma cells may result in melanocyte proliferation and melanin production $(22,34)$. However, these mechanisms were not evaluated in this study.

To gain more insight into the biological characteristics of these tumors, we performed immunohistochemical studies with p53, Ki-67, E-cadherin, and vimentin. The analyzed region was the invasive tumor front, which is the area of greatest tumor progression where the deepest and most aggressive cells reside (35). The inactivation and mutation of p53, which play an essential role in tumorigenesis and progression of head and neck carcinoma, are among the most frequent genetic alterations in oral squamous cell carcinoma (36). In oral malignancy, epithelial dysplastic lesion, and leukoplakia, p53 overexpression was identified via immunohistochemical studies (37-40). Similar to this finding, in all patients $(n=25)$, the proportion and staining intensity of positive p53 expression in the tumor was higher than that in the healthy epithelium. Current evidence regarding the prognostic value of p53 and $\mathrm{Ki}-67$ in patients with head and neck SCC is still controversial and inconclusive because of the heterogeneous and conflicting results $(25,41)$. Some studies found that p53 overexpression by more than $50 \%$ indicated a poor prognosis in advanced tumors, whereas a weak or negative expression indicated a good prognosis and longer survival (24). Some studies showed a significant relationship between $\mathrm{Ki}-67$ expression and overall survival, whereby, high $\mathrm{Ki}-67$ expression was a marker of poor prognosis in OSCC patients (25). Motta et al (42) reported that co-expression of p53 and Ki-67 markers in SCCs of the oral cavity and tongue suggested a worse prognosis. Despite the lack of significant differences between p53 and Ki-67 expression and clinicopathological parameters (data not shown), our study revealed that most POSCCs exhibited low expression of p53 and Ki-67. We found low expression of E-cadherin and positive expression of vimentin in only one tumor. Low expression of E-cadherin in OSCC has been previously reported to be associated with lower disease-free rates, higher rates of lymph node metastases, lower survival rates, and a more invasive histological pattern (29). Meanwhile, positive vimentin expression has been associated with a higher prevalence of recurrence and shorter survival time in OSCC and has been considered a reliable predictive EMT marker for poor prognosis in OSCC (43). Collectively, our immunohistochemical results suggest low malignancy of POSCC.

However, most of our understanding of oral melanocytes is derived from insights into the regulation and biology of epidermal melanocytes, which act not only to absorb ultraviolet radiation, but also as antioxidant and radical scavenging agents (44). Increasing evidence has shown that 
melanocytes are also active factors in the skin immune system and have immunomodulatory properties (45). In the oral cavity, melanocytes produced by the gingival epithelium can neutralize reactive oxygen species generated by dentogingival plaque-induced inflammation in the periodontal microenvironment (46). Moreover, treatment with antioxidants has the potential to prevent, inhibit, and reverse the multiple steps involved in oral carcinogenesis (47). Therefore, increased production of melanin with its antioxidant properties may be involved in the protective immune response and may inhibit the process of carcinogenesis in POSCC. In our study, intense inflammatory infiltration was observed near the pigmented area with localization of melanocytes in $21(84 \%)$ and at the nonpigmented area in $11(44 \%)$ patients. Hence, the localization of melanocytes is associated with inflammatory infiltration in POSCC. However, a firm conclusion about the association between melanocyte loading and immune response and their immunological significance in POSCC cannot be obtained due to the limited number of cases and evaluations. Thus, elucidation of the precise mechanism of this effect requires further studies with more specific molecular investigations.

This study has some limitations that might have influenced the results. Information regarding lifestyle habits was limited owing to the retrospective study design. In addition, we could not undermine the potential effects of previous mucosal diseases with post-inflammatory pigmentation. Our results need to be interpreted with caution from a non-comparative study. Future prospective investigations with a large cohort of patients and involving more specific molecular mechanisms are warranted to further extend our understanding of POSCC.

In conclusion, the clinical findings and immunohistochemical analyses presented here indicated that POSCC has a tendency towards nonaggressive oncological behavior and a good prognosis. These findings enhance our current understanding of the clinicopathological and biological characteristics of POSCC and further elucidate the oncological behavior of this rare pathological variant of OSCC.

\section{Acknowledgements}

The authors would like to thank Ms. Kiyoko Nagumo (Clinical Laboratory, Tokyo Medical and Dental University, Dental Hospital) for her technical support.

\section{Funding}

No funding was received.

\section{Availability of data and materials}

The datasets used and/or analyzed during the current study are available from the corresponding author on reasonable request.

\section{Authors' contributions}

$\mathrm{HH}$ and YM conceived the current study. TK and KK conceived and designed the experiments. $\mathrm{HH}$ and TK confirmed the authenticity of all the raw data. YO analyzed statistical data. TK interpreted the data and revised the manuscript. CMT was involved in all the stages of the study, performed the immunohistochemical staining and was a major contributor in writing the manuscript. All authors read and approved the final version of the manuscript.

\section{Ethics approval and consent to participate}

The study complied with the standards of the Declaration of Helsinki and was approved by the Institutional Review Board of Tokyo Medical and Dental University (approval no. D2020-600). The need for informed consent was waived owing to the retrospective nature of the study.

\section{Patient consent for publication}

Written informed consent for the publication of the oral photographs in Fig. 2 was obtained from the patients.

\section{Competing interests}

The authors declare that they have no competing interests.

\section{References}

1. Siegel RL, Miller KD and Jemal A: Cancer statistics, 2020. CA Cancer J Clin 70: 7-30, 2020.

2. Pulte D and Brenner H: Changes in survival in head and neck cancers in the late 20th and early 21st century: A period analysis. Oncologist 15: 994-1001, 2010.

3. Sim YC, Hwang JH and Ahn KM: Overall and disease-specific survival outcomes following primary surgery for oral squamous cell carcinoma: Analysis of consecutive 67 patients. J Korean Assoc Oral Maxillofac Surg 45: 83-90, 2019.

4. Iihara K, Yamaguchi K, Fujioka Y and Uno S: Pigmented neuroendocrine tumor of the lung, showing neuromelanin. Pathol Int 52: 734-739, 2002.

5. Zhang X, Liang Y and Wang HY: Invasive ductal carcinoma of the breast associated with extensive melanin melanosis: A case report and review of the literature. Int J Clin Exp Pathol 7: 1218-1223, 2014

6. Takeda Y and Kurose A: Pigmented mucoepidermoid carcinoma, a case report and review of the literature on melanin-pigmented salivary gland tumors. J Oral Sci 48: 253-256, 2006.

7. Satter EK: Pigmented squamous cell carcinoma. Am J Dermatopathol 29: 486-489, 2007.

8. Masuzawa N, Kishimoto M and Takahashi Y: Pigmented squamous cell carcinoma of the uterine cervix. Int J Gynecol Pathol 22: 285-288, 2003.

9. Shields JA, Shields CL, Eagle RC Jr, Singh AD, Demirci H and Wolf MA: Pigmented conjunctival squamous cell carcinoma simulating a conjunctival melanoma. Am J Ophthalmol 132: 104-106, 2001.

10. Mathews A, Abraham EK, Amman S and Nair MK: Pigmented squamous cell carcinoma of nasal cavity. Histopathology 33: 184-185, 1998.

11. Matsumoto M, Sonobe H, Takeuchi T, Furihata M, Iwata J, Ikeda $\mathrm{M}$ and Ohtsuki Y: Pigmented squamous cell carcinoma of the scrotum associated with a lentigo. Br J Dermatol 141: 132-136, 1999.

12. Kamiya M, Maehara R, Iizuka S, Yoshida T, Yamanouchi $H$, Yokoo H, Sasaki A, Hirato J and Nakazato Y: Pigmented squamous cell carcinoma with dendritic melanocyte colonization in the external auditory canal. Pathol Int 49: 909-912, 1999.

13. Patakas B,Hecker R and Kramer HS: Report on an oral, pigmented, squamous cell carcinoma. Int J Oral Surg 3: 445-448, 1974.

14. Ide F, Kusuhara S, Ohnuma H, Miyake T, Nakajima $T$ and Kimura T: Pigmented squamous cell carcinoma of the oral mucosa - with special reference to the role of non-keratinocytes in tumors and tumorous conditions. J Nihon Univ Sch Dent 23: $1-9,1981$.

15. Dunlap CL and Tomich CE: Melanocyte colonization of oral squamous cell carcinoma. Oral Surg Oral Med Oral Pathol 52: 524-530, 1981. 
16. Modica LA, Youngberg GA and Avila FO: Melanocyte colonization of an oral carcinoma. Histopathology 17: 477-478, 1990.

17. Kuwabara H, Uda H, Miyaguchi M, Nagai M, Saito K and Shibanushi T: Pigmented squamous cell carcinoma of the alveolar ridge in the oral mucosa. Oral Surg Oral Med Ora Pathol 77: 61-65, 1994.

18. Satomura K, Tokuyama R, Yamasaki Y, Yuasa T, Tatehara S, Ishimaru N, Hayashi Y and Nagayama M: Possible involvement of stem cell factor and endothelin-1 in the emergence of pigmented squamous cell carcinoma in oral mucosa. J Oral Pathol Med 36 621-624, 2007

19. Lisboa Castro J, Cazal C, Gomes Henriques AC, Carneiro Leão J, de Vasconcelos Carvalho M, de Carvalho Dourado HT and Carvalho AA: Pigmented oral squamous cell carcinoma: A case report and brief review of the literature. Int J Surg Pathol 17: $153-157,2009$.

20. Mikami T, Furuya I, Kumagai A, Furuuchi H, Hoshi H, Iijima S, Sugiyama Y and Takeda Y: Pigmented squamous cell carcinoma of oral mucosa: Clinicopathologic study of 3 cases. J Oral Maxillofac Surg 70: 1232-1239, 2012

21. Matsumoto N,Kitano T, Oki H, Omagari D, Matsue Y, Okudera M, Yamamura T, Nishikawa Y, Nishimura S, Asano M, et al: Pigmented oral carcinoma in situ: A case report and literature review. Oral Surg Oral Med Oral Pathol Oral Radiol 118: e79-e83, 2014.

22. Martins F, Mistro FZ, Kignel S, Palmieri M, do Canto AM and Braz-Silva PH: Pigmented squamous cell carcinoma in situ: Report of a new case and review of the literature. J Clin Exp Dent 9: e1362-e1365, 2017

23. Zhou G, Liu Z and Myers JN: TP53 Mutations in head and neck squamous cell carcinoma and their impact on disease progression and treatment response. J Cell Biochem 117: 2682-2692, 2016.

24. Cutilli T, Leocata P, Dolo V and Altobelli E: p53 as a prognostic marker associated with the risk of mortality for oral squamous cell carcinoma. Oncol Lett 12: 1046-1050, 2016

25. Xie S, Liu Y, Qiao X, Hua RX, Wang K, Shan XF and Cai ZG: What is the prognostic significance of $\mathrm{Ki}-67$ positivity in ora squamous cell carcinoma? J Cancer 7: 758-767, 2016.

26. Wan Y, Liu H, Zhang M, Huang Z, Zhou H, Zhu Y, Tao Y, Xie N Liu X, Hou J, et al: Prognostic value of epithelial-mesenchymal transition-inducing transcription factors in head and neck squamous cell carcinoma: A meta-analysis. Head Neck 42 $1067-1076,2020$

27. Sobin LH, Gospodarowicz MK and Wittekind C (eds): TNM Classification of Malignant Tumours. John Wiley \& Sons, Ltd. West Sussex, p332, 2010.

28. Anneroth G, Batsakis J and Luna M: Review of the literature and a recommended system of malignancy grading in oral squamous cell carcinomas. Scand J Dent Res 95: 229-249, 1987.

29. Costa LC, Leite CF, Cardoso SV, Loyola AM, Faria PR, Souza PE and Horta MC: Expression of epithelial-mesenchymal transition markers at the invasive front of oral squamous cell carcinoma. J Appl Oral Sci 23: 169-178, 2015.

30. Shen $\mathrm{H}$ and $\mathrm{Wu} \mathrm{W}$ : Study of melanin bleaching after immunohistochemistry of melanin-containing tissues. Appl Immunohistochem Mol Morphol 23: 303-307, 2015.

31. Yu CH, Chen HH, Liu CM, Jeng YM, Wang JT, Wang YP, Liu BY, Sun A and Chiang CP: HMB-45 may be a more sensitive maker than S-100 or Melan-A for immunohistochemical diagnosis of primary oral and nasal mucosal melanomas. J Oral Pathol Med 34: 540-545, 2005
32. De Silva RK, Siriwardena BSMS, Samaranayaka A, Abeyasinghe WAMUL and Tilakaratne WM: A model to predict nodal metastasis in patients with oral squamous cell carcinoma. PLoS One 13: e0201755, 2018.

33. Abidullah M, Kiran G, Gaddikeri K, Raghoji S and Ravishankar TS: Leuloplakia - review of a potentially malignant disorder. J Clin Diagn Res 8: ZE01-ZE04, 2014.

34. Hirobe T: Role of keratinocyte-derived factors involved in regulating the proliferation and differentiation of mammalian epidermal melanocytes. Pigment Cell Res 18: 2-12, 2005.

35. Sharma M, Sah P, Sharma SS and Radhakrishnan R: Molecular changes in invasive front of oral cancer. J Oral Maxillofac Pathol 17: 240-247, 2013.

36. Li Y, Li B, Xu B, Han B, Xia H, Chen QM and Li LJ: Expression of $\mathrm{p} 53, \mathrm{p} 21$ (CIP1/WAF1) and eIF4E in the adjacent tissues of oral squamous cell carcinoma: Establishing the molecular boundary and a cancer progression model. Int J Oral Sci 7: 161-168, 2015.

37. Varun BR, Ranganathan K, Rao UK and Joshua E: Immunohistochemical detection of p53 and p63 in oral squamous cell carcinoma, oral leukoplakia, and oral submucous fibrosis. J Investig Clin Dent 5: 214-219, 2014.

38. Swaminathan U, Joshua E, Rao UK and Ranganathan $K$ : Expression of p53 and Cyclin D1 in oral squamous cell carcinoma and normal mucosa: An Immunohistochemical study. J Oral Maxillofac Pathol 16: 172-177, 2012.

39. Lavieille JP, Brambilla E, Riva-Lavieille C, Reyt E, Charachon R and Brambilla C: Immunohistochemical detection of $\mathrm{p} 53$ protein in preneoplastic lesions and squamous cell carcinoma of the head and neck. Acta Otolaryngol 115: 334-339, 1995.

40. Nadal A, Campo E, Pinto J, Mallofré C, Palacín A, Arias C, Traserra $\mathbf{J}$ and Cardesa A: p53 expression in normal, dysplastic, and neoplastic laryngeal epithelium. Absence of a correlation with prognostic factors. J Pathol 175: 181-188, 1995.

41. Tandon S, Tudur-Smith C, Riley RD, Boyd MT and Jones TM: A systematic review of p53 as a prognostic factor of survival in squamous cell carcinoma of the four main anatomical subsites of the head and neck. Cancer Epidemiol Biomarkers Prev 19: 574-587, 2010.

42. Motta RR, Zettler CG, Cambruzzi E, Jotz GP and Berni RB: Ki-67 and p53 correlation prognostic value in squamous cell carcinomas of the oral cavity and tongue. Rev Bras Otorrinolaringol (Engl Ed) 75: 544-549, 2009.

43. Liu S, Liu L, Ye W, Ye D, Wang T, Guo W, Liao Y, Xu D, Song H, Zhang L, et al: High vimentin expression associated with lymph node metastasis and predicated a poor prognosis in oral squamous cell carcinoma. Sci Rep 6: 38834, 2016.

44. Feller L, Masilana A, Khammissa RA, Altini M, Jadwat $Y$ and Lemmer J: Melanin: The biophysiology of oral melanocytes and physiological oral pigmentation. Head Face Med 10: 8, 2014.

45. Plonka PM, Passeron T, Brenner M, Tobin DJ, Shibahara S, Thomas A, Slominski A, Kadekaro AL, Hershkovitz D, Peters E, et al: What are melanocytes really doing all day long...? Exp Dermatol 18: 799-819, 2009.

46. Nilima S and Vandana KL: Melanin: A scavenger in gingival inflammation. Indian J Dent Res 22: 38-43, 2011

47. Choudhari SK, Chaudhary M, Gadbail AR, Sharma A and Tekade S: Oxidative and antioxidative mechanisms in oral cancer and precancer: A review. Oral Oncol 50: 10-18, 2014.

(i) (3) This work is licensed under a Creative Commons Attribution-NonCommercial-NoDerivatives 4.0 International (CC BY-NC-ND 4.0) License. 